\title{
Experiencia con el uso del colgajo en voltereta en pacientes del Hospital del Trabajador de Santiago: una alternativa válida*
}

\author{
Drs. JUAN JOSÉ LOMBARDI A. ${ }^{1}$, CRISTIÁN ARRIAGADA I. ${ }^{2}$, DANIEL CALDERÓN M. ${ }^{2}$, \\ WILFREDO CALDERÓN O. ${ }^{2}$, CLAUDIO GUERRA S. ${ }^{2}$
}

1 Residente de Cirugía General. Universidad de Los Andes.

2 Servicio de Cirugía. Hospital del Trabajador.

Santiago, Chile.

\begin{abstract}
Experience using turn over flap in patients at the Hospital del Trabajador de Santiago: a valid alternative

Background: The plastic surgeon is frequently faced with need of choosing from a variety of local and distant flaps for the coverage of difficult wounds. In 1981, Thatte described a local flap consisting in a random pattern deepithelialized flap adjacent to the lesion rotated in $180^{\circ}$ with a dermoepidermal graft to cover the flap and donating zone. Objective: To present the experience of the Plastic Surgery and Burns Unit of the Hospital del Trabajador de Santiago with the use of this flap. Material: Retrospective study of 16 Turn Over flaps in 15 patients. Results: The complications recorded were 1 case of graft loss and 2 partial lost of the flap. We didn't observe complications directly related to the flap. Conclusions: This type of flap is another therapeutic alternative in certain cases. Rate Base: Length $=1: 2$ is appropriate to ensure irrigation.
\end{abstract}

Key words: Surgical flaps, reconstructive surgical procedures, turnover flap.

\section{Resumen}

Frecuentemente el cirujano plástico se enfrenta a la necesidad de utilizar colgajos para lograr cubrir una serie de defectos de cobertura cutánea. En 1981, Thatte describió una nueva técnica de colgajo, la que consistía en el levantamiento de un colgajo random desepitelizado, anexo a la lesión, y posteriormente doblarlo sobre su base, para finalmente ser injertado junto con la zona dadora. Nuestro objetivo es presentar la experiencia del Servicio de Cirugía Plástica y Quemados del Hospital del Trabajador de Santiago con el uso de este colgajo. Se revisó en forma retrospectiva el resultado de 16 colgajos en 15 pacientes realizados con esta técnica. Se observó en 1 caso pérdida del injerto y en 2 casos pérdida parcial del colgajo en voltereta. Durante el seguimiento no se observaron complicaciones directamente relacionadas con el colgajo. Nos parece que este tipo de colgajo es una alternativa válida en determinados casos. La proporción base: longitud = 1: 2 es adecuada para asegurar su irrigación

Palabras clave: Colgajos quirúrgicos, cirugía reconstructiva, colgajo en voltereta.

*Recibido el 9 de junio de 2010 y aceptado para publicación el 10 de enero de 2011.

Correspondencia: Dr. Juan José Lombardi A.

Camino del Ayuntamiento 2006, Santiago, Chile.

juanjoselombardi@gmail.com 


\section{Introducción}

Frecuentemente el cirujano plástico se enfrenta a la necesidad de utilizar colgajos para lograr cubrir una serie de defectos. En la mayoría de los casos se opta por colgajos tradicionales, como el axial, tipo random, en isla y libres ${ }^{1}$. Sin embargo, la realización de este tipo de colgajos no es siempre posible, ya sea por la complejidad de la lesión, por la escasez de tejido local o por la necesidad de un procedimiento rápido.

En 1981, Thatte, publicó una nueva técnica de colgajo la que consistía en el levantamiento de un colgajo tipo random desespitelizado previamente, anexo a la lesión, y posteriormente doblarlo sobre su base (quedando la superficie desepitelizada hacia la profundidad, cubriendo la lesión, y la parte dermograsa del colgajo hacia la superficie) para finalmente ser injertado junto con la zona dadora. Este colgajo recibió el nombre de colgajo turn over ${ }^{2}$, que en lengua castellana se traduce como en voltereta (Figura 1). Este tipo de colgajo se basa en la doble irrigación dada por las anastomosis intradermales de los plexos dérmicos y subdérmicos.

Posterior a su publicación, el colgajo en voltereta se ha utilizado para el tratamiento de diversas lesiones ya sea en extremidades superiores ${ }^{3}$, inferiores, abdomen, tórax, cabeza y cuello ${ }^{4}$, e incluso para la cobertura en pacientes quemados 5 , con resultados bastante alentadores tanto a corto como a largo plazo. Pese a esto, este colgajo ha sido bastante criticado en la literatura con la base de que tendría una alta probabilidad de pérdida dada su relativa insuficiente irrigación.

Nuestro objetivo es describir los casos de cobertura con este colgajo y presentar la experiencia del Hospital del Trabajador de Santiago (HTS).

\section{Material y Método}

\section{Selección de pacientes}

Se analizó en forma retrospectiva el sistema de archivos del Hospital del Trabajador de Santiago entre los años 2003 y 2009, seleccionando aquellos en los cuales se realizó algún procedimiento rotulado como colgajo (Fasciocutaneo o Axial de Pie, Fasciocutaneo o Axial de Pierna, Fasciocutáneo o Axial de Muslo, Fasciocutáneo o Axial Braquial, Cutáneo de Transposición), revisándose un total de 414 fichas. Posteriormente se seleccionó aquellos protocolos en los cuales se describiera la utilización del colgajo en turn over obteniéndose finalmente un total de 15 casos seleccionables.

Se describen las características de los pacientes y su diagnóstico, además se presenta el tiempo operatorio y de seguimiento junto con las complicaciones durante la hospitalización y durante el seguimiento.

\section{Técnica quirúrgica}

Como se observa en forma esquemática en la Figura 1, la técnica consiste en el levantamiento de un colgajo dermograso, previamente desepitelizado, dibujado en alguna zona adyacente a la lesión. Una vez levantado el colgajo es doblado sobre su base la cual funciona como un pivote. De esta manera la zona desepitelizada queda mirando hacia la lesión (lo cual resulta beneficioso ya que la zona desepitelizada tiene muy buena vascularización ${ }^{2}$ ) y la zona dermograsa hacia la superficie (Figuras 2a, 2b y 3). Finalmente se realiza la cobertura con un injerto dermoepidérmico (Figura 4).

Es fundamental asegurar una buena irrigación de este colgajo, la cual estará dada finalmente por su base. En este sentido la relación Base: Longuitud del colgajo utilizada fue de 1:2 con una base dese-

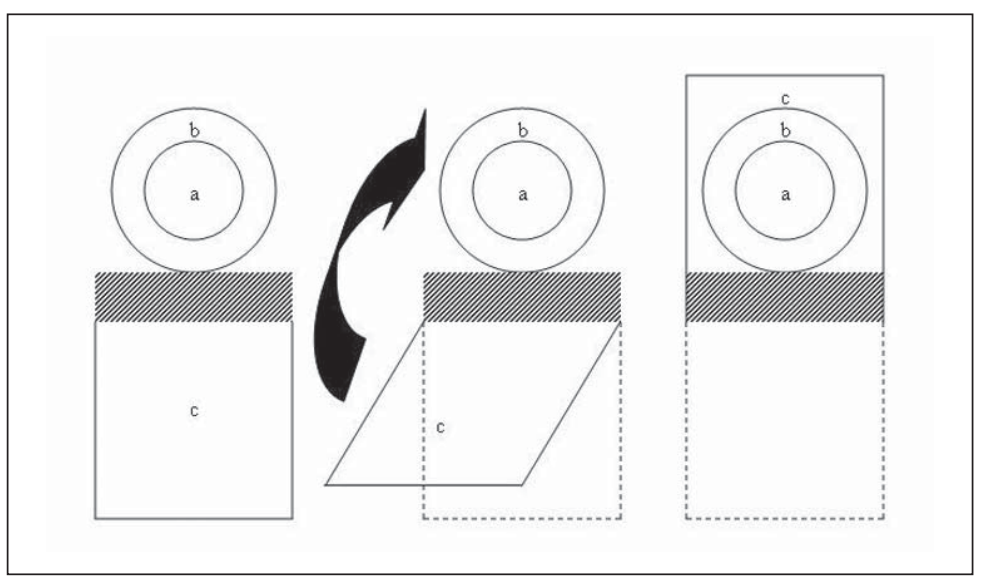

Figura 1. Técnica quirúrgica en forma esquemática. El defecto presenta la zona a (zona de exposición ósea o tendinea) y zona b (tejido subcutáneo circundante). La zona achurada representa la base del colgajo que aporta la irrigación del mismo. El colgajo está representado por la letra c. Se realiza el levantamiento del colgajo el cual se dobla sobre sí mismo (como abriendo un libro) para finalmente cubrir el defecto (zonas a y b) así como también su base (zona achurada). 


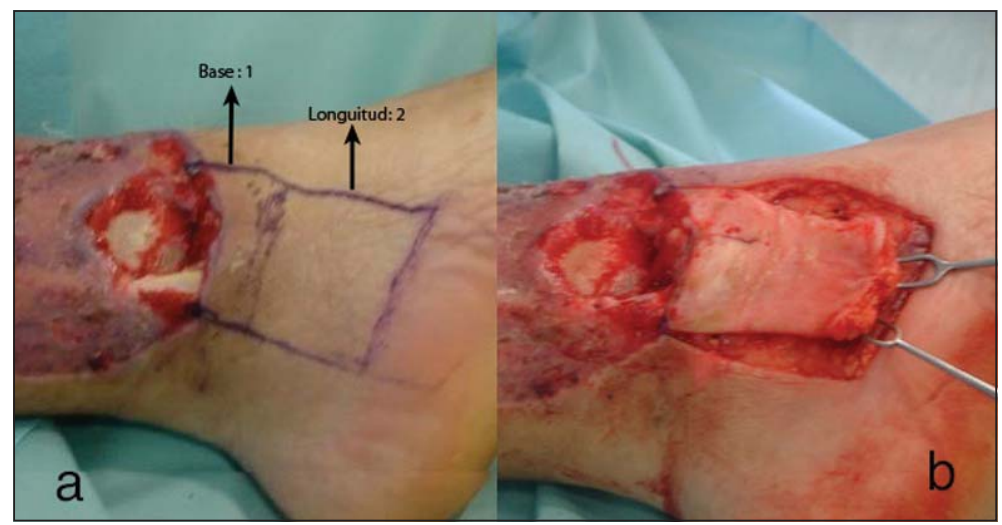

Figura 2. Paciente de 39 años de edad que sufre desforramiento de su pierna izquierda con exposición del maléolo medial y compromiso tendineo. Se muestra el diseño del colgajo para su cobertura (relación 1:2) (a). Levantamiento del colgajo (aún no desepitelizado) (b).

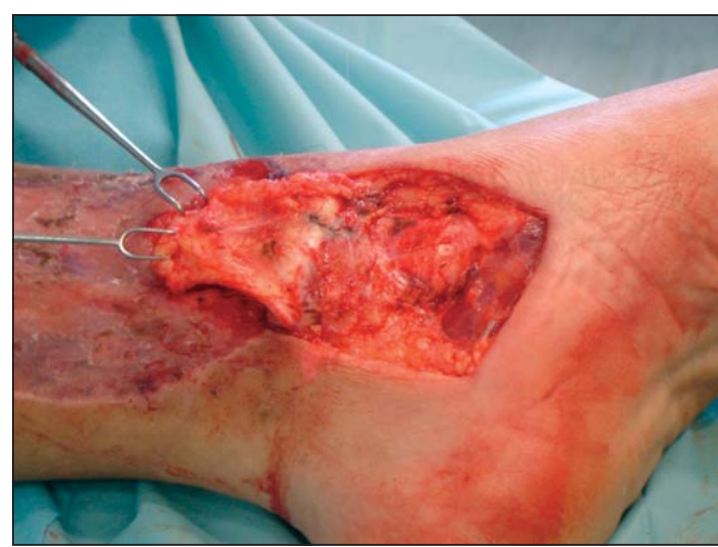

Figura 3. Doblaje del colgajo, como la hoja de un libro, quedando cubierto tanto el defecto como la base del colgajo.

pitelizada no menor a 1,5 cms de ancho. La relación clásicamente descrita es de 1:36 y en algunas publicaciones se decribe la no desepitelización de la zona de la base del colgajo argumentando que esto protejería aún más la irrigación ${ }^{4}$. ción.

La base del colgajo será la que aporte su irriga-

En resumen, todos nuestros colgajos fueron dermograsos, relación 1:2, base mínimo de 1,5 cms de ancho, y con una base desepitelizada.

\section{Resultados}

En el período analizado se realizaron un total de 16 colgajos en 15 pacientes (14 hombres y 1 mujer). La edad fluctuó entre 25 y los 63 años.

Se realizaron 10 colgajos en la extremidad inferior, 3 en la extremidad superior, y 3 en cabeza y cuello, para cubrir diversos defectos (Tabla 1).

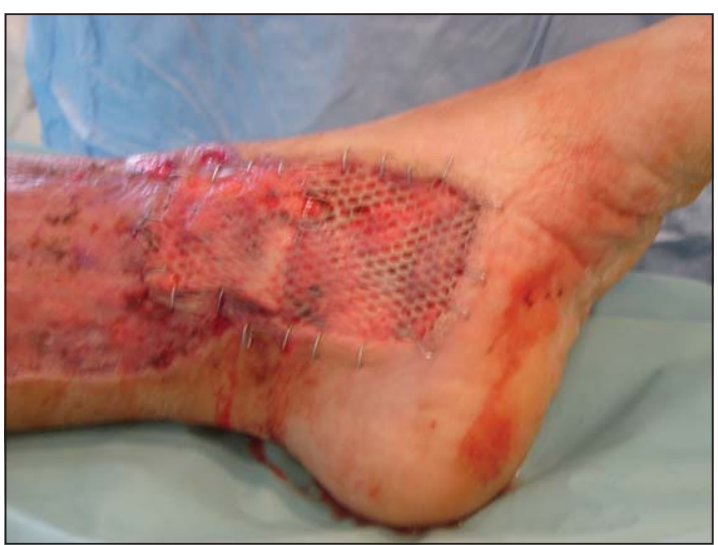

Figura 4. Injerto Dermo Epidérmico (IDE) que cubre la zona dadora y el colgajo.

Tabla 1. Diagnóstico y cantidad de pacientes tratados con colgajo en voltereta

\begin{tabular}{lc}
\hline n Pacientes & 15 \\
Cantidad de colgajos & 16 \\
Extremidad inferior & 10 \\
$\quad$ Desforramiento pierna & 1 \\
Fractura tobillo expuesta & 2 \\
$\quad$ Fractura pierna expuesta & 3 \\
$\quad$ Úlcera cutánea de pierna post traumática & 4 \\
Extremidad superior & 3 \\
$\quad$ Quemadura eléctrica manos & 1 \\
$\quad$ Quemadura y herida de mano complicada & 2 \\
Cabeza y cuello & 3 \\
$\quad$ Carcinoma basocelular & 1 \\
Linfoma nasal & 1 \\
$\quad$ Quemadura eléctrica nasal & 1 \\
\hline
\end{tabular}




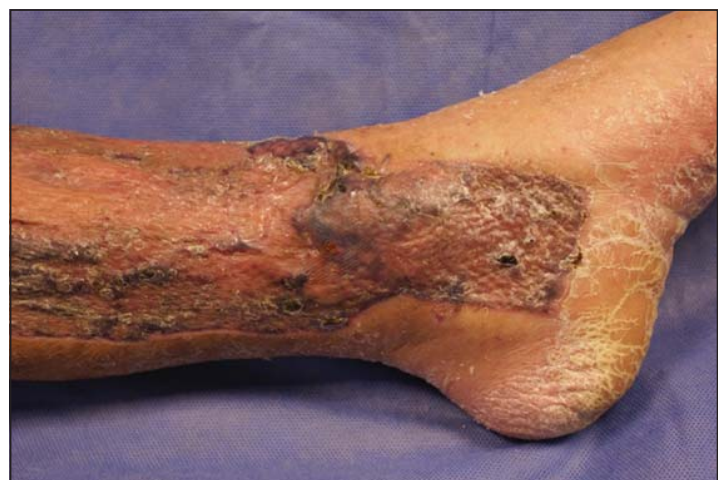

Figura 5. Resultado post operatorio.

El tiempo operatorio fue entre 30 y 180 minutos (promedio 80 minutos), y el tiempo de seguimiento fue entre 3 y 18 meses.

En cuanto a las complicaciones postoperatorias durante la hospitalización, éstas se presentaron en 3 pacientes. En 1 caso, con diagnóstico de úlcera residual del muñón por amputación traumática del primer ortejo, se requirió un nuevo injerto, y 2 casos, con diagnósticos de úlcera cutánea post traumática en tercio distal de la pierna y fractura expuesta de tibia, presentaron pérdida parcial del colgajo en voltereta. En el primer paciente se realizó un colgajo sural, el cual resulta una alternativa válida de reparación para defectos del tercio distal de la pierna y que ha sido descrito como alternativa ante la falla de otros colgajos ${ }^{7}$ y en el segundo un colgajo de avance.

Durante el seguimiento no se observaron complicaciones directamente relacionadas con el colgajo. En 1 paciente, con diagnóstico de úlcera post traumática en la pierna, se presentó un quiste de inclusión (probablemente secundario a la sutura), el cual se manejó en forma ambulatoria con drenaje y curaciones, evolucionando en forma favorable. Un segundo paciente, en el que se realizó un colgajo sural, se presentó una fístula del borde del colgajo, la cual se manejó con curaciones en forma ambulatoria con buenos resultados. Ninguno de estos pacientes requirió rehospitalización.

Al término del estudio todos los pacientes se encontraban de alta y en buenas condiciones.

\section{Discusión}

Si bien el colgajo en voltereta no es utilizado en forma frecuente, nos parece una muy buena alternativa frente a diversas lesiones y situaciones.

En nuestros pacientes, la mayor indicación fue en lesiones de las extremidades inferiores y en particular en su tercio distal (Figuras 2 a 5). Esto coincide

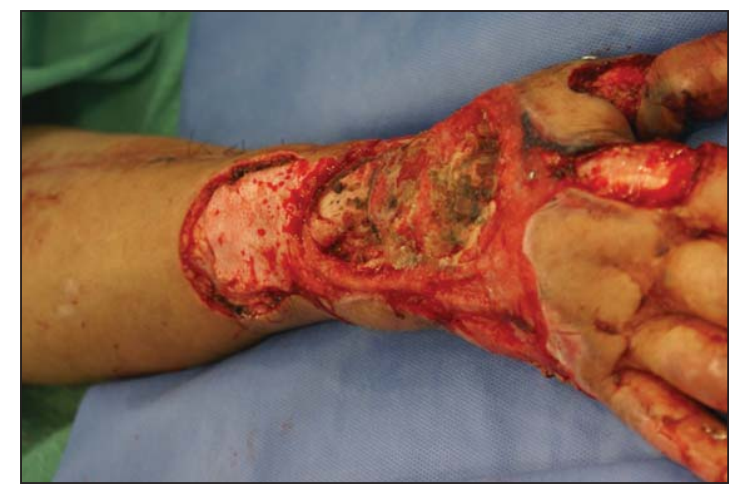

Figura 6. Paciente de 38 años de edad que sufre quemadura eléctrica en mano derecha con puerta de salida en pies. Presenta exposición ósea. Se decide cobertura con colgajo en voltereta. Diseño y levantamiento del colgajo con base proximal a la lesión.

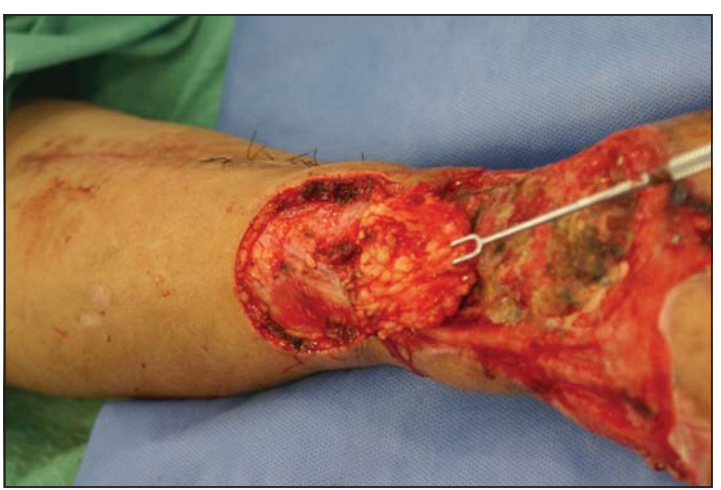

Figura 7. Giro (voltereta) del colgajo y cobertura de la exposición ósea.

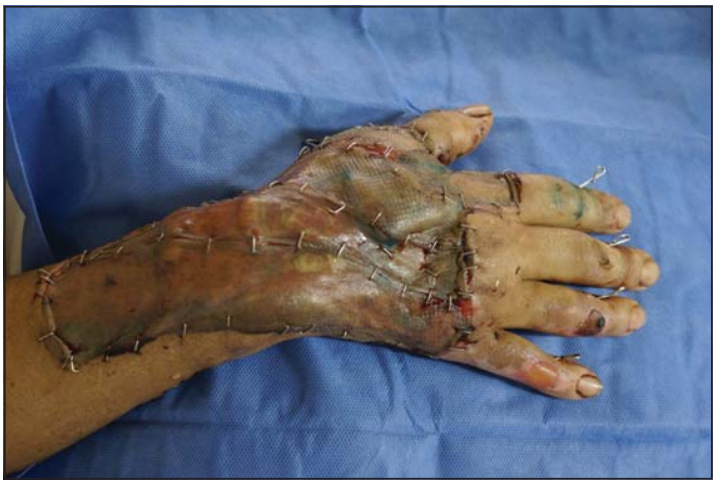

Figura 8. Cobertura con IDE.

con las aplicaciones en su descripción inicial en $1981^{2}$. Hemos visto que algunos autores lo utilizan como primera opción en defectos pequeños de tobillo, heridas en pacientes con enfermedad vascular terminal, y heridas con exposición del tendón de Aquiles ${ }^{4}$. 
Además de lo anterior se utilizó la técnica en otras zonas o situaciones donde su uso no es frecuente, como cara, pacientes quemados (Figuras 6 a 8) y en extremidad superior, con buenos resultados tanto a corto como a largo plazo ${ }^{3-8}$.

Otra de las ventajas que presenta este colgajo, es la corta duración del procedimiento, que se realiza en un solo tiempo quirúrgico, aportando una buena cobertura. Además, para un mismo defecto, podrían utilizarse colgajos provenientes de varias zonas locales adyacentes a la lesión ${ }^{4}$.

Una de las críticas a este colgajo es su patrón de irrigación. En nuestros casos no se presentó ningún sufrimiento del colgajo atribuible al patrón de irrigación manteniendo una relación base: longitud de 1:2, que aseguraría su viabilidad, en contraposición a lo previamente publicado en la cual describen una irrigación límite pues usan una relación 1:34.

Sólo en 2 pacientes hubo pérdida marginal del colgajo y 1 paciente requirió un nuevo injerto. Dado lo anterior nos parece que la técnica es segura cuando se cumplen las proporciones propuestas y cuando está bien indicado.

Todos nuestros pacientes fueron colgajos tipo random en los cuales, al realizar la voltereta, la porción desepitelizada quedaba en contacto con la lesión y la grasa, con su rico plexo dérmico y subdérmico la cual era injertada. En nuestra casuística no incorporamos colgajos fascio-grasos, los que han sido usados como una variación relativa de la técni$\mathrm{ca}^{4-6}$. En este contexto cabe mencionar publicaciones con estudios en cadáveres en los que se ha estudiado la irrigación de la arteria dorsal del pie para la cobertura, con este tipo de colgajos, de defectos en el antepié. En ellos se ha demostrado que se obtiene una muy buena irrigación con dimensiones de 1:3,5 hasta 1:6,5 de relación base: colgajo ${ }^{9}$. Hay que tener en cuenta que lo anterior asegura una mejor irrigación ya que funcionaría como un colgajo pediculado, lo cual permite la utilización de dimensiones más grandes.

Finalmente, basándonos en nuestra experiencia, nos parece que el colgajo en voltereta es una opción válida para el tratamiento de diversas lesiones, en especial aquellas del tercio distal de la extremidad inferior y en aquellas zonas en que la disponibilidad de tejidos es baja y su irrigación es límite. Es una técnica aplicable cuando no se dispone de tejidos donantes locales y cuando no se desea dejar grandes defectos en la zona dadora.

Si bien nuestra muestra es pequeña, creemos que esta experiencia podría alentar a otros grupos a realizar este tipo de colgajos, disponiendo así de una herramienta más para el tratamiento de nuestros pacientes, especialmente en aquellos con patologías agregadas (diabetes, ateroesclerosis, alteraciones inmunitarias, etc.), y en pacientes de mayor edad, en los cuales la irrigación es defectuosa.

\section{Referencias}

1. Calderón W. Colgajos Fascio cutáneos en extremidad inferior. Cuad Chil Cir. 1990;32:384-9.

2. Thatte RL. Random-pattern de-epithelialised "turn over" flaps to replace skin loss in the upper third of the leg. $\mathrm{Br}$ J Plast Surg. 1981;34:312-4.

3. Thatte R L, Gopalakrishna A, Prasad S. The use of deepithelialised "turn over" flap in the hand. Br J Plast Surg. 1982;35:293-9.

4. Mitra A, Spears J, Newsome E, Mc Campbell B, Kiran R, Mitra A. Expanding the scope of the turnover flap. Plast Reconstr Surg. 2006;118:125-33.

5. Ramakrishnan K, Jayaraman V, Ramachandran K, Mathivanan T. Deepithelialized turnover flaps in burns. Plast Reconstr Surg. 1988;82:262-6.

6. Thatte RL. Deepithelialized “Turnover” Skin Flap of the Lower Leg and Foot. En: Strauch B, Vasconez L.O, Hall-Findlay E.J, Lee B.T, editors. Grabb’s Encyclopedia of Skin Flaps: Torso, Pelvis, and Lower Extremities, $3^{\mathrm{a}}$ ed. Philadelphia: Editorial: Lippincott Williams \& Wilkins; 2009:1452-7.

7. Calderón W, Cabello R, Covarrubias P, Parada F, Piña J, Anziani F, y col. Reconstrucción de lesiones de extremidad inferior con Colgajo de Arteria Sural Superficial, experiencia de 10 años. Rev Chil Cir. 2007;59:132-5.

8. Al-Qattan M. The use of adipofascial turnover flaps for coverage of complex dorsal ring finger defects caused by electric burns. Burns 2005;31:643-6.

9. El-Khatib, Hamdy A. Adipofascial turn-over flap based on perforators of the dorsalis pedis for resurfacing forefoot defects: an anatomic and clinical approach. Plast Reconstr Surg. 1998;102:393-7. 